\title{
Orbital Sarcoma cM1 TNM Finding v8
}

National Cancer Institute

\section{Source}

National Cancer Institute. Orbital Sarcoma CM1 TNM Finding v8. NCI Thesaurus. Code C140836.

Orbital sarcoma with distant metastasis. (from AJCC 8th Ed.) 